\title{
Evaluation of a genus reactive monoclonal antibody in rapid identification of Chlamydia trachomatis by direct immunofluorescence
}

\author{
I ALEXANDER,* I D PAUL, † AND E O CAUL† \\ From the *Department of Genitourinary Medicine, Bristol Royal Infirmary, and the + Public Health \\ Laboratory, Kingsdown, Bristol
}

SUMMARY This study compared the direct visualisation of elementary bodies in urogenital smears by the direct immunofluorescence test with the isolation of Chlamydia trachomatis in McCoy cell cultures treated with cytochalasin B.

$C$ trachomatis was isolated from $41(16 \cdot 4 \%)$ of 250 unselected specimens in McCoy cell cultures treated with cytochalasin B. Of the 41 culture positive specimens $37(90 \%)$ were diagnosed by direct visualisation of elementary bodies in the smears using the direct immunofluorescence test. Four specimens were positive by isolation only and a further seven were positive by the direct immunofluorescence test only. Overall the direct immunofluorescence test had a scnsitivity of $90 \%$ and a specificity of $97 \%$.

The presence of red blood cells or mucus or the use of barrier creams did not appear to interfere with the normal visualisation of elementary bodies using the direct immunofluorescence test.

\section{Introduction}

The role of Chlamydia trachomatis as a pathogen in the genital tract has been clearly defined, ${ }^{\prime}$ and it is now considered to be the most common sexually transmitted agent in the Western world. ${ }^{2}$ However, the establishment of a comprehensive, nationally based service for confirming the diagnosis of a genital chlamydial infection in the United Kingdom remains a major problem. At present the identification of $C$ trachomatis relies on cell culture because the detection of chlamydial antibodies in serum, although of value in establishing the aetiology of infertility, ${ }^{34}$ is not a reliable indication of an uncomplicated current infection in the general population. ${ }^{5}$ The laboratory diagnosis of infection with $C$ trachomatis is considered to be time consuming and expensive in terms of initial capital outlay, and has consequently been restricted to a few major centres. Specimens transported to central laboratories from peripheral units often fail to give reliable results unless the strict criteria for sampling and transport can be met. ${ }^{6} \mathrm{At}$ present, attempts to provide a culture service for districts or in many regions of the United Kingdom is considered to be uneconomic.

Address for reprints: Dr E O Caul, Public Health I aboratory, Myrtle Road, Kingsdown, Bristol BS2 8EL

Accepted for publication 19 November 1984
An inexpensive, reproducible, rapid diagnostic technique is required to detect $C$ trachomatis. This study has evaluated a direct genus specific immunofluorescence test (Boots Celltech Diagnostics Ltd, Slough, Berkshire, England) in detecting chlamydiae in urogenital smears. This test was compared with the culture on McCoy cells of $C$ trachomatis from urogenital specimens taken from unselected men and women patients attending the department of genitourinary medicine at the Bristol Royal Infirmary.

\section{Patients, materials, and methods}

\section{CLINICAL EXAMINATIONS}

We screened 250 unselected patients (137 men, 113 women) seen consecutively by IA for evidence of infection with $C$ trachomatis. Endocervical swabs from women patients and urethral swabs (inserted at least two inches into the urethra) from men patients were collected for chlamydial culture. These swabs were rolled on to $6 \mathrm{~mm}$ wells of teflon coated slides before being placed into transport medium ${ }^{7}$ for chlamydial isolation. The smears were air dried before being fixed in acetone for five minutes. All specimens were transported within three hours of collection to the Public Health I.aboratory for processing.

During the course of the clinical examination we enquired about any recent or current medication, 
especially with antibiotics. Other factors were taken into consideration such as contraceptive practice, use of barrier spermicidal preparation, and the presence of blood on the cervix or endocervical canal caused by sampling or frank menstruation.

\section{LABORATORY METHODS}

\section{Isolation of chlamydiae}

McCoy cell cultures treated with cytochalasin B were used to isolate $C$ trachomatis as described previously. ${ }^{6}$

\section{Immunofluorescence}

Urethral and cervical smears previously fixed in acetone were coded, and $25 \mu \mathrm{l}$ of direct immunofluorescence test reagent (monoclonal antibody specific to the genus Chlamydia conjugated with fluorescein isothiocyanate) was added to each well. One well per specimen was tested, and slides were incubated for 15 minutes at $37^{\circ} \mathrm{C}$ in a moist chamber. At the end of the incubation period the slides were briefly rinsed in tap water before being agitated gently for five minutes in a container of distilled water. Slides were air dried and mounted in glycerol containing a photobleaching inhibitor. All preparations were examined separately by two of us (EOC and IDP) using incident blue light fluorescence at $\times 1000$ magnification. Specimens containing ten or more evenly distributed "elementary bodies" were considered positive. A positive control consisting of elementary bodies disrupted from McCoy infected cultures were included in each day's batch. When all the results were obtained, the specimens were decoded for comparison with the cell culture results.

\section{Results}

\section{APPEARANCE OF CHLAMYDIA ANTIGEN IN SMEARS}

Positive smears that contained more than 1000 elementary bodies, which were spherical and uniform in size, also contained large numbers of brightly fluorescing particulate bodies, which were about one third of the size of the elementary bodies. Such smears also often contained apparently disrupted large inclusions, which also stained intensely.

In contrast, positive smears containing small numbers of elementary bodies (10-100) did not appear to have these brightly fluorescing particulate bodies or disrupted inclusions.

CULTURE AND DIRECT IMMUNOFLUORESCENCE The table shows that $C$ trachomatis was isolated in McCoy cell cultures treated with cytochalasin B from $41(16 \cdot 4 \%)$ of 250 specimens from unselected patients. Of the 41 culture positive specimens, 37
TABLE Analysis of results using direct immunofluorescence and culture on McCoy cells of specimens from 250 patients

\begin{tabular}{llllcr}
\hline Results of: & & & \multicolumn{3}{c}{ No of specimens from: } \\
\cline { 1 - 1 } \cline { 5 - 6 } Culture & Immunofluorescence & & Men & Women & Total \\
\hline Positive & Positive & 22 & 15 & 37 \\
Positive & Negative & 2 & 2 & 4 \\
Negative & Positive & 5 & 2 & 7 \\
Negative & Negative & 108 & 94 & 202 \\
Total & & 137 & 113 & 250 \\
\hline
\end{tabular}

$(90 \%)$ were diagnosed by direct visualisation of elementary bodies in the smears using the direct immunofluorescence test. The four culture positive and immunofluorescence negative specimens contained $0,1,3$, and 4 elementary bodies respectively, and a further seven smears were positive by the direct immunofluorescence test. The presence of red blood cells or mucus or the use of barrier creams did not appear to interfere with the normal visualisation of elementary bodies using the direct immunofluorescence test.

\section{Discussion}

This study showed that the direct visualisation of elementary bodies in smears using the direct immunofluorescence test was comparable with isolating chlamydia in McCoy cell cultures treated with cytochalasin B, which are currently used routinely in our laboratory. Overall the direct immunofluorescence test had a sensitivity of $90 \%$ and a specificity of $97 \%$.

At the time of the study reported here 10 or more elementary bodies in a smear using a prototype genus reactive monoclonal antibody supplied by Boots Celltech were considered to be diagnostic of a current chlamydial infection. Some positive specimens containing large numbers of elementary bodies also contained copious amounts of brightly fluorescing particulate bodies. Such small particulate bodies may possibly represent the glycolipid, genus reactive, heat stable antigen. Further studies are necessary to clarify this observation. Some negative smears $(<10$ elementary bodies) contained a few brightly fluorescing apple green particles of the same order of size as elementary bodies, but which were not associated with cells. This "grey area" has now been resolved by the commercially available chlamydial test reagent, Imagen.

In our experience, the detection of inclusions in McCoy cell cultures treated with cytochalasin B was more rapid than the detection of elementary bodies in smears when such elementary bodies were present in low numbers $(<20)$. Equally, negative cultures could be examined more rapidly than negative smears 
stained by the direct immunofluorescence test. Smears containing large numbers of elementary bodies, however, which often also contained partially disrupted brightly fluorescing inclusions, were readily detected. Such rapid diagnosis may not, however, generally be required. The direct immunofluorescence test will be invaluable in the rapid diagnosis of neonatal and adult oculogenital infections, when treatment can be started rapidly. This technique can also be used to enable early treatment of genital chlamydial infection in women undergoing termination or spontaneous abortion.

We think that laboratories examining less than $\mathbf{3 0}$ smears a day could use this test to advantage. In our experience, however, it becomes tiring to examine more than this number in a session.

Finally, it became apparent with increasing experience that the diagnosis of chlamydial infection based on the direct immunofluorescence test became much easier when elementary bodies were associated with cells. Further studies are now in progress to assess the lowest number of elementary bodies detectable before a definitive diagnosis of infection with chlamydiae can be made. Provisional results suggest that the commercially available Imagen chlamydial test is excellent for detecting small numbers of elementary bodies (five or six cell associated elementary bodies per smear) when adequate experience has been gained. This observation partly agrees with recently reported studies using a monoclonal antibody of different specificity, in which even smaller numbers of elementary bodies have been considered to be diagnostic of a chlamydial infection. ${ }^{89}$

The Imagen chlamydial immunofluorescence test has considerable potential as a technique for diagnosing genital infection. Specimens can be collected, air dried, and fixed in acetone before they are transported to the laboratory. This overcomes the logistical difficulties associated with current culture technique. The greatest impact will be for departments of genitourinary medicine that are at present without any diagnostic service, and other specialties needing a similar diagnostic facility. Laboratories that have already committed capital outlay to establishing culture services and training staff may be less likely to see the need for monoclonal techniques, apart from rapid diagnosis of certain infections, such as neonatal and adult oculogenital conjunctivitis. However, in laboratories that provide a diagnostic service for outlying clinics, which experience problems in the transport of samples, the monoclonal system would be a valuable alternative to culture.

We thank Drs A N McClean and P Taylor for their cooperation in allowing access to their patients.

\section{References}

1. Schachter J. Chlamydial infections (first of three parts). $N$ Engl J Med 1978; 298:248-435.

2. Dunlop EMC. Chlamydial genital infection and its complications. Br J Hosp Med 1983;29:6-11.

3. Conway D, Glazener CMA, Caul EO, et al. Chlamydial serology in fertile and infertile women. Lancet 1984; i: 191-3.

4. Moore DE, Spadoni LR, Foy HM, et al. Increased frequency of serum antibodies to Chlamydia trachomatis in infertility due to distal tubal disease. Lancet 1982; ii: 574-7.

5. Richmond SJ, Caul EO. Fluorescent antibody studies in chlamydial infections. J Clin Microbiol 1975; 1:345-52.

6. Richmond SJ, Paul ID, Taylor PK. Value and feasibility of screening women attending STD clinics for cervical chlamydial infections. British Journal of Venereal Diseases 1980;56:92-5.

7. Richmond SJ. The isolation of chlamydia subgroup A (Chlamydia trachomatis) in irradiated McCoy cells. Medical Laboratory Technology 1974; 31:7-9.

8. Thomas BJ, Evans RT, Hawkins DA, Taylor-Robinson D. Sensitivity of detecting Chlamydia trachomatis elementary bodies in smears by use of a fluorescein labelled monoclonal antibody: comparison with conventional chlamydial isolation. J Clin Pathol 1984;37:812-6.

9. Tam MR, Stamm WE, Handsfield HH, et al. Culture-independent diagnosis of Chlamydia trachomatis using monoclonal antibodies. N Engl J Med 1984; 310:1146-50. 